Noninvasive nuclear magnetic resonance profiling of painting layers

Federica Presciutti, Juan Perlo, Federico Casanova, Stefan Glöggler, Costanza Miliani, Bernhard Blümich, Brunetto Giovanni Brunetti, and Antonio Sgamellotti

Citation: Appl. Phys. Lett. 93, 033505 (2008);

View online: https://doi.org/10.1063/1.2963026

View Table of Contents: http://aip.scitation.org/toc/apl/93/3

Published by the American Institute of Physics

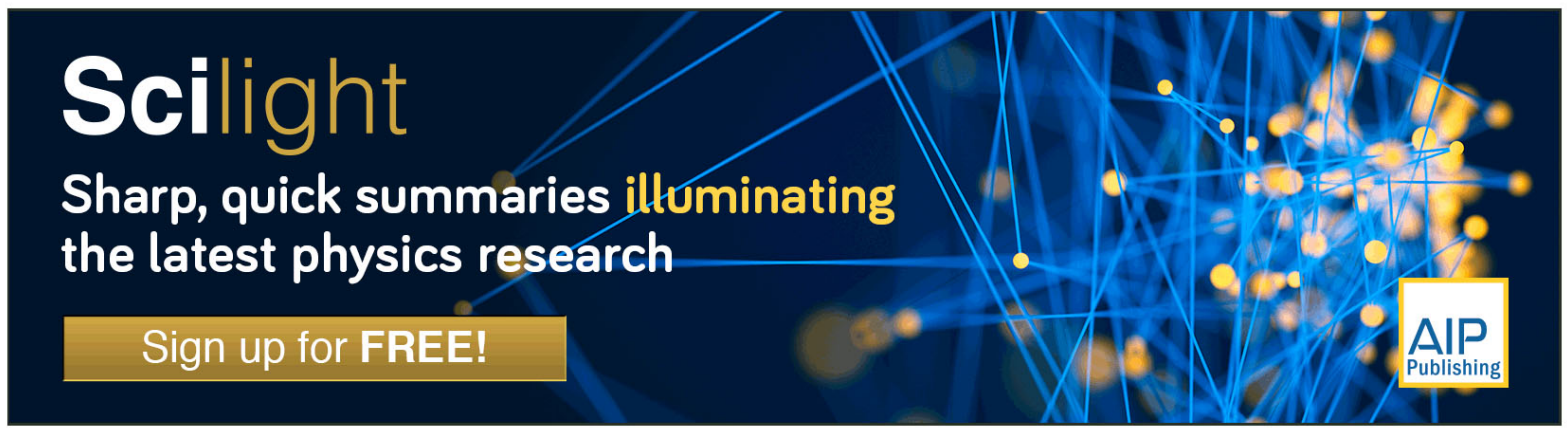




\title{
Noninvasive nuclear magnetic resonance profiling of painting layers
}

\author{
Federica Presciutti, ${ }^{1, a)}$ Juan Perlo, ${ }^{2}$ Federico Casanova, ${ }^{2}$ Stefan Glöggler, ${ }^{2}$ \\ Costanza Miliani, ${ }^{1}$ Bernhard Blümich, ${ }^{2}$ Brunetto Giovanni Brunetti, ${ }^{1}$ and \\ Antonio Sgamellotti ${ }^{1}$ \\ ${ }^{1}$ Centre of Excellence SMAArt and ISTM-CNR, clo Chemistry Department, University of Perugia, \\ Via Elce di Sotto 8, Perugia 06123, Italy \\ ${ }^{2}$ Institut für Technische Chemie und Makromolekulare Chemie, Rheinisch-Westfälische Technische \\ Hochschule, Aachen D-52056, Germany
}

(Received 16 April 2008; accepted 1 July 2008; published online 22 July 2008)

\begin{abstract}
In this work we demonstrate the potential of single-sided nuclear magnetic resonance (NMR) sensors to access deeper layers of paintings noninvasively by means of high-resolution depth profiles spanning several millimeters. The performance of the sensor in resolving painting structures was tested on models for which excellent agreement with microscopy techniques was obtained. The depth profiling NMR technique was used in situ to investigate old master paintings. The observation of differences in NMR relaxation times of tempera binders from these paintings and from artificially aged panels raises the possibility to differentiate between original and recently restored areas. (C) 2008 American Institute of Physics. [DOI: 10.1063/1.2963026]
\end{abstract}

Paintings are composed of multiple layers, such as preparation, underdrawing, paint, varnish, etc. The layer structure, or stratigraphy, provides useful information as it reflects the working practices and techniques of the artist. Conservation and restoration require precise knowledge of constituent materials and their complex multilayer structures. As the objects are precious and often cannot be moved, the appropriate diagnostic techniques have to be ideally noninvasive and should be carried out in situ by portable instrumentation. Elemental and molecular information can be retrieved noninvasively in situ by spectroscopic techniques such as X-ray fluorescence, fiber optic Fourier transform infrared (FTIR), fiber optic micro-Raman, and others, but these techniques only provide characterization of materials near the surface of the paint without in-depth information. ${ }^{1-3}$

For a stratigraphic analysis today it is still common practice to extract small samples to obtain the cross section of the paint for subsequent examination by optical microscopy (OM) and electron microscopy (SEM), micro-FTIR, microRaman spectroscopy, and other techniques. ${ }^{4}$ Such studies allow conservators and scholars to visualize the sequence of layers in the paint and to potentially identify pigments and binders, possible repaintings, the imprimitura (the primer), and also the presence of an incamottatura, a textile used to cover and join the wood panels of the support as a base for the primer.

As such studies are compromising to the painting, we investigated the use of single-sided nuclear magnetic resonance (NMR) as an alternative method. ${ }^{5-10}$ A single-sided NMR sensor with a flat sensitive volume external to the device ${ }^{10}$ can provide profiles of the stratigraphy of paintings and other nontransparent objects spanning several millimeters in depth with a resolution greater than $10 \mu \mathrm{m}$, which are sensitive to the physical-chemical properties of the materials. ${ }^{11}$ Competing techniques that can also provide stratigraphic information noninvasively include optical coherence tomography and ultrasound, but these are also still

\footnotetext{
${ }^{a)}$ Electronic mail: federica@thch.unipg.it.
}

experimental, and sometimes cannot penetrate deeper into the layer structure than the varnish layer. ${ }^{12}$

This work reports the results obtained with the Profile NMR-MOUSE $^{10,11}$ (Mobile Universal Surface Explorer by ACT GmbH, Roetgen, Germany), a compact and mobile, single-sided, ${ }^{1} \mathrm{H}$ NMR sensor. It has been applied to measure in situ the thickness of proton-rich layers in paintings and to investigate binder aging, without contact with the paint surface. This investigation is a first step toward characterizing the three dimensional (3D) morphology of the different layers in paintings without contact and as such avoiding any kind of sampling.

The NMR-MOUSE is a mobile NMR sensor equipped with a single-sided permanent magnet arrangement that generates a uniform static magnetic field gradient suitable for high-resolution sample profiling up to depths of about $10 \mathrm{~mm}$ from objects placed nearby. Its size is approximately $100 \times 100 \times 80 \mathrm{~mm}^{3}$. The NMR signal derives from a thin slice centered $10 \mathrm{~mm}$ above the device parallel to its surface, where the field strength is $0.411 \mathrm{~T}$ and the gradient is $20 \mathrm{~T} / \mathrm{m}$ along the depth direction. The slice volume extends about $10 \times 10 \mathrm{~mm}^{2}$ along the lateral directions, a size that is determined by the geometry of the radio-frequency coil of the sensor, while the thickness in the depth direction depends on the setting of the measurement parameters and can range from 200 to $2.3 \mu \mathrm{m}$.

The sensor is mounted on a stepper-motor driven lift, which can be positioned with a precision of $10 \mu \mathrm{m}$ to scan the profile by shifting the sensitive slice through the object. For each slice the transverse magnetization decay is recorded with a multiecho train by CPMG (Carr, Purcell, Meiboom, and Gill) sequence. The measured signal is reduced to a $T_{2}$-weighted proton density value of the material within the sensitive slice by partial integration of the echo envelope. The collection of these numbers as a function of depth forms the measured NMR depth profile. $T_{1}$ weighting can also be introduced to the measurement by varying the recycle delay between subsequent acquisitions. By default, the recycle delay is chosen large enough to avoid $T_{1}$ weighting. The profile amplitude at each depth was computed from the sum of the 

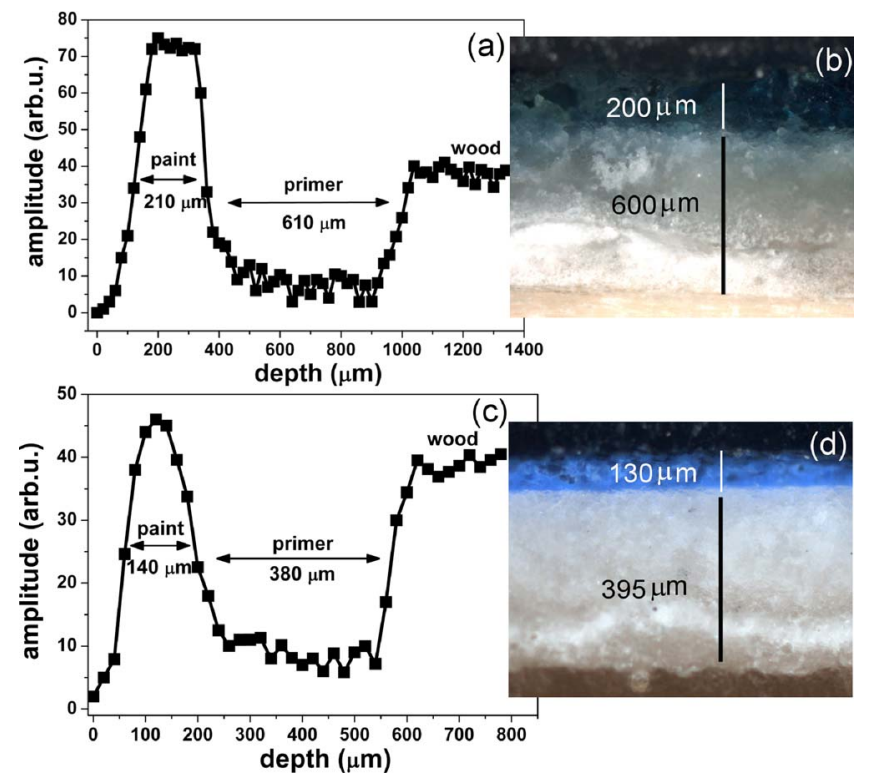

FIG. 1. (Color online) Depth profiles collected for the two easel painting models (top: panel 1 with copper acetate as pigment; bottom: panel 2 with cobalt blue as pigment). The CPMG sequence was used to collect transverse magnetization decays with an echo time $t_{E}=0.062 \mathrm{~ms}, t_{R}=0.1 \mathrm{~s}$, and 1024 scans, leading to a measuring time about $100 \mathrm{~s}$ per point in the profile. The depth profiles $a$ and $c$ were measured with a resolution of $30 \mu \mathrm{m}$. Images $b$ and $d$ across the paint layers were obtained with an optical microscope on sample cross sections removed invasively from the panels.

maxima of the first echoes in the acquired echo train.

The suitability and accuracy of the method were previously tested on two easel painting models which had been prepared at the University of Perugia approximately 5 years ago following the recipes of the old masters. They consisted of a wood panel covered by a primer composed of gypsum and animal glue, and finally a paint layer containing two different pigments mixed with egg tempera, a common binder in ancient paintings. In one panel, the pigment was verdigris, copper acetate $\left[\mathrm{Cu}\left(\mathrm{CH}_{3} \mathrm{COO}\right)_{2} \mathrm{H}_{2} \mathrm{O}\right]$, in the other it was cobalt blue $\left[\mathrm{Co} \cdot \mathrm{Al}_{2} \mathrm{O}_{4}\right]$. To demonstrate the performance of the NMR sensor, depth profiles of the two models were measured and compared to OM and SEM cross sections.

Depth profiles of two laboratory models were measured with a resolution of $30 \mu \mathrm{m}$ (Fig. 1). The profiles [Figs. 1(a) and 1(c)] show amplitude variations which can be assigned
TABLE I. Layer thicknesses of the two easel painting models by SEM and Profile NMR-MOUSE.

\begin{tabular}{lcc}
\hline \hline & $\begin{array}{c}\text { SEM } \\
(\mu \mathrm{m})\end{array}$ & $\begin{array}{c}\text { NMR-MOUSE } \\
(\mu \mathrm{m})\end{array}$ \\
\hline Panel 1, primer & $600 \pm 48$ & $610 \pm 20$ \\
Panel 1, paint layer & $200 \pm 12$ & $210 \pm 20$ \\
Panel 2, primer & $395 \pm 28$ & $380 \pm 20$ \\
Panel 2, paint layer & $130 \pm 10$ & $140 \pm 20$ \\
\hline \hline
\end{tabular}

to the different layers of the paintings. Starting from the surface of the painting, the two regions of higher amplitude correspond to the paint layer and the wood, whereas the intermediary zone is ascribable to the primer, as confirmed by the optical images of the cross section of the models [Figs. 1(b) and 1(d)]. Thus, due to the different properties of the constituent materials, the NMR profiles indeed reveal the stratigraphy of the paintings. The thickness of the layers was determined by measuring the distance from the position of half maximum amplitude of the first edge to the equivalent point of the second edge. The values for the paint layer are $210 \pm 20 \mu \mathrm{m}$ for panel 1 and $140 \pm 20 \mu \mathrm{m}$ for panel 2 .

To check the validity of the NMR results, electron and OM measurements of the thickness of the layers were carried out on cross sections obtained invasively by sampling the same area of the panel as investigated by the NMR-MOUSE. The values obtained for panels 1 and 2 are $200 \pm 12$ and $130 \pm 10 \mu \mathrm{m}$, respectively [Figs. 1(b) and 1(d)]. The SEM values result from an average of ten measurements done on a sample $\left(10 \times 5 \times 2 \mathrm{~mm}^{3}\right)$ taken from the area investigated by the NMR probe, and the error corresponds to the standard deviation. In comparison, the error in the thickness from the NMR profiles was determined as the standard deviation calculated from a number of measurements performed at the same spot. The measured thickness values of the different layers in the models using both noncontact NMR and invasive microscopy techniques are summarized in Table I. The results show very good agreement.

The same NMR sensor was then used to measure a large number of Italian Renaissance master paintings in the Galleria Nazionale dell'Umbria in Perugia (Italy). As an example, two depth profiles measured at different points on an oil painting, the "Adorazione dei Magi" $(\sim 1470)$ by Pietro Vannucci ("il Perugino"), are reported in Fig. 2. Four different
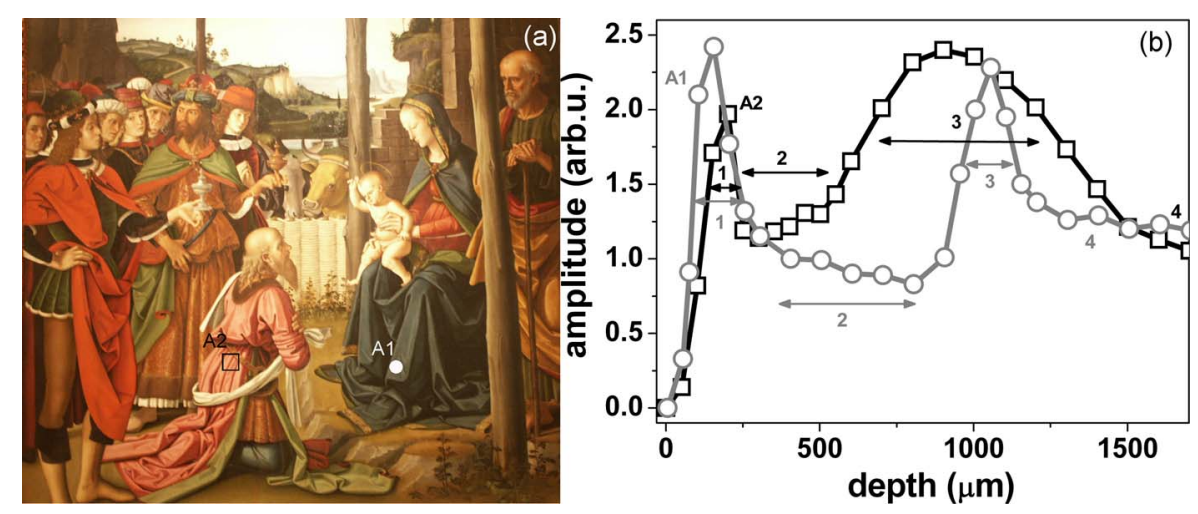

FIG. 2. (Color online) Depth profiles recorded at the two positions indicated in the Adorazione dei Magi by il Perugino $(\sim 1470)$ with a spatial resolution of $50 \mu \mathrm{m}$. The profiles were derived from summing the first four echoes of a CPMG echo train collected with an echo time $t_{E}=0.034 \mathrm{~ms}$, a recycle delay $t_{R}$ $=0.1 \mathrm{~s}$, and 512 scans. The measuring time for a whole profile was of about $30 \mathrm{~min}$. The thickness of the layers collected in the area A1 and A2 are the following: 1 (paint layer)=140 and $100 \mu \mathrm{m}, 2$ (imprimitura) $=600$ and $400 \mu \mathrm{m}, 3$ (canvas and glue) $=200$ and $620 \mu \mathrm{m}$, and 4 is the wood of the panel. 
layers were observed. They are ascribed to the wood, the incamottatura, the imprimitura and the paint layer. No varnish layer was detected. The canvas of the incamottatura glued to the wood is around $200 \mu \mathrm{m}$ thick at one position (A1) and about three times $(620 \mu \mathrm{m})$ that value at another position (A2), where two boards were joined. The thickness of the primer layer is about $600 \mu \mathrm{m}$ in A1 and $400 \mu \mathrm{m}$ in A2. The paint layers is around $140 \mu \mathrm{m}$ thick in A1, while in A2 is thinner and differs of about $40 \mu \mathrm{m}$. These results clearly show that the technique is suitable for measuring the thickness of the paint layer, the imprimitura, and the incamottatura in historical paintings with an accuracy of about 20-30 $\mu \mathrm{m}$ without any contact with the painting's surface. The measurements are simple, and the instrumentation is easily portable and therefore can be used directly at the site where the artwork is located. In particular, it is possible to detect the presence and thickness of canvas reinforcements, a task not easily achieved by other noninvasive methods (Fig. 2).

With this technique it is possible to scan entire paintings with depth profiles acquired point by point to construct a 3D map of the stratigraphy. Moreover, the technique can in principle be also applied to the study of the nature of the binder (i.e., oil, tempera, casein, etc.) used for binding the pigments. In particular, the aging of the binders can be investigated by measuring the NMR relaxation times $T_{1}$ and $T_{2}$ as they characterize the molecular mobility, which may be related to their aging history. To compare the values obtained from the old master paintings (A), the relaxation times were also measured for a number of different tempera paints prepared at the National Gallery in London and artificially aged in incremental steps by repeated exposure to intense light irradiation (B). In a $T_{1}-T_{2}$ correlation plot (Fig. 3) the values for naturally aged paints from the old master paintings show lower relaxation times than the values for the more recent and artificially aged paint samples. This shows that different stages of tempera aging, or different aging conditions, can be discriminated by the NMR method, indicating that NMR has the potential to differentiate between old and recently restored sections in a painting thus raising the hope of identifying genuine and forged paintings.

This work was carried out within EU-ARTECH, an Integrated Infrastructures Initiative supported by the 6th Framework Programme of the European Union (Contract

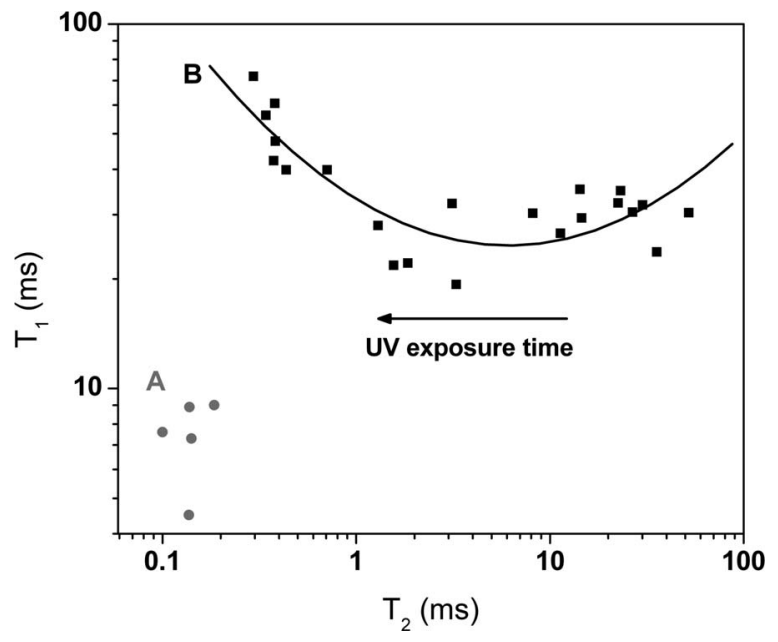

FIG. 3. Natural (A) and artificial aging (B) of tempera colors. Intense light aging reduces $T_{2}$ but not $T_{1}$, while natural aging over several centuries reduces $T_{1}$ and $T_{2}$.

\section{RII3-CT-2004-506171).}

The authors wish to thank Marika Spring and Ashok Roy of the Scientific Department of the National Gallery of London who kindly offered for analysis the artificially aged panel.

${ }^{1}$ C. Miliani, F. Rosi, I. Borgia, P. Benedetti, B. G. Brunetti, and A. Sgamellotti, Appl. Spectrosc. 61, 293 (2007).

${ }^{2}$ C. Ricci, C. Miliani, B. G. Brunetti, and A. Sgamellotti, Talanta 69, 1221 (2006).

${ }^{3}$ C. Miliani, F. Rosi, A. Burnstock, B. G. Brunetti, and A. Sgamellotti, Appl. Phys. A: Mater. Sci. Process. 89, 849 (2007).

${ }^{4}$ Modern Analytical Methods in Art and Archaeology, edited by E. Ciliberto and G. Spoto (Wiley, New York, 2000).

${ }^{5}$ R. L. Kleinberg, in Encyclopedia of NMR, edited by D. M. Grant and R. K. Harris (Wiley, New York, 1996), p. 4960.

${ }^{6}$ G. A. Matzkanin, in Nondestructive Characterization of Materials, edited by P. Höller et al. (Springer, Berlin, 1989), p. 655.

${ }^{7}$ G. Eidmann, R. Salvelsberg, P. Blümler, and B. Blümich, J. Magn. Reson., Ser. A 122, 104 (1996).

${ }^{8}$ B. Blümich, S. Anferova, S. Sharma, A. L. Segre, and C. Federici, J. Magn. Reson. 161, 204 (2003).

${ }^{9}$ N. Proietti, D. Capitani, E. Pedemonte, B. Blümich, and A. L. Segre, J. Magn. Reson. 170, 113 (2004).

${ }^{10}$ J. Perlo, F. Casanova, and B. Blümich, J. Magn. Reson. 176, 64 (2005).

${ }^{11}$ B. Blümich, Essential NMR (Springer, Berlin, 2005).

${ }^{12}$ T. Arecchi, M. Bellini, C. Corsi, R. Fontana, M. Materazzi, L. Pezzati, and A. Tortora, Opt. Spectrosc. 101, 23 (2006). 